\title{
The dominance, covering and supercovering relations in the context of multiple partially specified reciprocal relations
}

\author{
Raúl Pérez-Fernández, Irene Díaz, Susana Montes and Bernard De Baets
}

\begin{abstract}
The problem of ranking different candidates or alternatives according to the preferences of different voters or experts is a common study subject in the fields of social choice theory and preference modelling. Whereas the former field normally restricts its attention to preferences given in the form of rankings (with ties), the latter field embraces the use of (partially specified) reciprocal relations. In this contribution, we study the notions of dominance relation, covering relation and supercovering relation, which are widely studied in the setting in which we are dealing with rankings (with ties), and adapt them to the setting in which we are dealing with partially specified reciprocal relations by using a tool similar to stochastic dominance.
\end{abstract}

Keywords: Reciprocal relation; Dominance; Covering; Supercovering.

Raúl Pérez-Fernández

Department of Statistics and O.R. and Mathematics Didactics, University of Oviedo, Spain

KERMIT, Department of Data Analysis and Mathematical Modelling, Ghent University, Belgium

e-mail: perezfernandez@uniovi.es; raul.perezfernandez@ugent.be

Irene Díaz

Department of Informatics, University of Oviedo, Spain

e-mail: sirene@uniovi.es

Susana Montes

Department of Statistics and O.R. and Mathematics Didactics, University of Oviedo, Spain

e-mail: montes@uniovi.es

Bernard De Baets

KERMIT, Department of Data Analysis and Mathematical Modelling, Ghent University, Belgium e-mail: bernard.debaets@ugent.be 


\section{Introduction}

The aggregation of preferences (on a set of candidates) given in the form of a list of rankings has been a popular topic in the field of social choice theory for centuries [6,9]. This field has witnessed its greatest period of splendor since the appearence of Arrow's impossibility theorem [1,2], which states that there is no method for aggregating a given list of rankings into a single ranking in a way that satisfies some desirable properties. One should note that Arrow's impossibility theorem only arises in the presence of three or more candidates since in the case of two candidates the simple majority rule $[11,17,21,31]^{1}$ fulfills the aforementioned desirable properties. Unfortunately, in case three or more candidates are considered, it is known that the simple majority rule might yield a cyclical relation - a phenomenon usually referred to as the Condorcet paradox - and, thus, lead to Arrow's impossibility theorem.

Once it is established there is no perfect method for the aggregation of rankings, different study subjects arise. For instance, we could take a totally different approach and abandon the aggregation of rankings by asking the voters to evaluate the candidates individually, thus obtaining an aggregated utility value for each candidate, which could be used for ranking all the candidates. This problem has been addressed by many authors, especially in the context of bargaining [23], decision making [24] and voting $[3,4]$. If one would like to stick to the problem of the aggregation of rankings, one could focus on the proposal of different methods fulfilling other desirable properties not listed by Arrow (e.g. the method of Kemeny [19] can be interpreted as a maximum-likelihood estimator of a true unobservable ranking [34] or the method of ranked pairs [33, 35] and the method of Schulze [30] have been proved to be independent of clones). Another option is to admit as a possible outcome of the aggregation any possible binary relation. For instance, one could simply consider the dominance relation given by the simple majority rule, which amounts to computing the (smallest) median relation [5]. More elaborate options are the covering relation [22] and the supercovering relation [25], which are typically quite sparse.

Nobel-prize laureate Sen [32] expressed: "it is certainly arguable that what matters is not merely the number who prefer $x$ to $y$ and the number who prefer $y$ to $x$, but also by how much each prefers one alternative to the other". In this direction, the field of (fuzzy) preference modelling $[13,29]$ has also built on some similar foundations as social choice theory, while now allowing the voters to express different intensities of preference in terms of, for instance, (valued) reciprocal relations or linguistic labels. Prominent notions in social choice theory have been analysed in the setting in which voters express different intensities of preference, e.g., the simple majority rule [14], the Borda count [16], approval voting [15] and the covering relation [28].

\footnotetext{
${ }^{1}$ We recall that a candidate is said to defeat another candidate by simple majority if the number of voters who prefer the former candidate to the latter one is greater than the number of voters who prefer the latter candidate to the former one.
} 
In this paper, we follow the direction started in [27] and discuss the setting in which each voter expresses a partially specified reciprocal relation. In particular, we recall the most important notions from [27] in Section 2 and discuss how the covering relation and the supercovering relation could be defined in this setting in Section 3. We end with some conclusions in Section 4.

\section{Preliminaries}

We consider the problem setting in which $r$ voters/experts ${ }^{2}$ are asked to compare $k$ candidates/alternatives ${ }^{2}$ and the goal is to obtain a collective comparison of the different candidates (preferably by ranking them from best to worst). In our particular problem setting, each voter expresses his/her own personal partially specified reciprocal relation $[7,10,18]$ on the set of candidates $\mathscr{C}$. This means that, for at least one couple of candidates, but not necessarily for all possible couples of candidates, the voter expresses a value in the unit interval ${ }^{3}$. The value 0.5 represents indifference between two candidates and the closer to 1 (resp. 0), the stronger the degree of preference of the first (resp. second) candidate over the second (resp. first) one. It is assumed that every two candidates are compared by at least one voter. In that way, each voter expresses a relation $P^{\ell}: \mathscr{C}^{2} \rightarrow[0,1]$ where, for any $\ell \in\{1, \ldots, r\}$ and any $\left(a_{i}, a_{j}\right) \in \mathscr{C}^{2}$, it holds that $P^{\ell}\left(a_{i}, a_{j}\right)+P^{\ell}\left(a_{j}, a_{i}\right)=1$ or that both $P^{\ell}\left(a_{i}, a_{j}\right)$ and $P^{\ell}\left(a_{j}, a_{i}\right)$ are undefined. By convention, $P^{\ell}\left(a_{i}, a_{i}\right)$ is considered undefined for any $\ell \in\{1, \ldots, r\}$ and any $a_{i} \in \mathscr{C}$. The fact that an element $P^{\ell}\left(a_{i}, a_{j}\right)$ is undefined is denoted by $P^{\ell}\left(a_{i}, a_{j}\right)=\emptyset$. For any $\ell \in\{1, \ldots, r\}$ and any $a_{i}, a_{j} \in \mathscr{C}, P^{\ell}\left(a_{i}, a_{j}\right)$ is referred to as the degree of preference of the $\ell$-th voter for candidate $a_{i}$ over candidate $a_{j}$.

Given the partially specified reciprocal relations $\left(P^{\ell}\right)_{\ell=1}^{r}$ given by the voters, we denote by $D\left(a_{i}, a_{j}\right)$ the multi-set of all degrees of preference expressed for a candidate $a_{i}$ over a candidate $a_{j}$, i.e.,

$$
D\left(a_{i}, a_{j}\right)=\left\{P^{\ell}\left(a_{i}, a_{j}\right) \mid \ell \in\{1, \ldots, r\} \wedge P^{\ell}\left(a_{i}, a_{j}\right) \neq \emptyset\right\} .
$$

We refer to $D\left(a_{i}, a_{j}\right)$ as the preference distribution for candidate $a_{i}$ over candidate $a_{j}$ or, equivalently, as the preference distribution for the couple $\left(a_{i}, a_{j}\right)$. Multi-sets, which are sets of elements where duplicated elements are allowed, are denoted with double curly brackets \{\}$\}$.

Example 1. Consider the set $\mathscr{C}=\{a, b, c, d\}$ of $k=4$ candidates and the following partially specified reciprocal relations given by $r=4$ voters, represented as matrices

\footnotetext{
2 The terms 'voter' and 'candidate' are commonly used in the field of social choice, whereas the terms 'expert' and 'alternative' are more extended in the field of preference modelling. We stick to the former notation throughout the remainder of this paper.

${ }^{3}$ Admittedly, a voter should not be expected to provide a large variety of values in the unit interval. However, a broad range of values might be a result of a prior process for making uniform the information provided by the different voters [8].
} 
$P^{\ell}$, where the element at the $i$-th row and the $j$-th column represents the degree of preference of the $\ell$-th voter for the $i$-th candidate over the $j$-th candidate (candidates are indexed in alphabetical order):

$$
\begin{aligned}
& P^{1}=\left(\begin{array}{cccc}
\emptyset & 0.6 & 1 & \emptyset \\
0.4 & \emptyset & 0.4 & \emptyset \\
0 & 0.6 & \emptyset & 0.6 \\
\emptyset & \emptyset & 0.4 & \emptyset
\end{array}\right), \quad P^{2}=\left(\begin{array}{cccc}
\emptyset & 0.7 & \emptyset & 1 \\
0.3 & \emptyset & \emptyset & 0.4 \\
\emptyset & \emptyset & \emptyset & \emptyset \\
0 & 0.6 & \emptyset & \emptyset
\end{array}\right), \\
& P^{3}=\left(\begin{array}{cccc}
\emptyset & \emptyset & \emptyset & 1 \\
\emptyset & \emptyset & 0.8 & \emptyset \\
\emptyset & 0.2 & \emptyset & 0.6 \\
0 & \emptyset & 0.4 & \emptyset
\end{array}\right), \quad \quad P^{4}=\left(\begin{array}{cccc}
\emptyset & \emptyset & \emptyset & 1 \\
\emptyset & \emptyset & \emptyset & \emptyset \\
\emptyset & \emptyset & 0 & 0.7 \\
0 & \emptyset & 0.3 & \emptyset
\end{array}\right) .
\end{aligned}
$$

For the couple $(a, b)$, the first voter has expressed a degree of preference of 0.6 for $a$ over $b$ (thus, 0.4 for $b$ over $a$ ), whereas the second voter has expressed a degree of preference of 0.7 for $a$ over $b$ (thus, 0.3 for $b$ over $a$ ). Note that the third voter and the fourth voter have not expressed any preference for $a$ over $b$ (nor for $b$ over $a$ ). Therefore, the preference distribution for $a$ over $b$ is the multi-set $D(a, b)=\{0.6,0.7\}$. Since we are dealing with reciprocal relations, the preference distribution for $b$ over $a$ is the multi-set $D(b, a)=\{0.3,0.4\}$. We can calculate the preference distribution of any couple in $\mathscr{C}_{\neq}^{2}=\left\{\left(a, a^{\prime}\right) \in \mathscr{C}^{2} \mid a \neq a^{\prime}\right\}$ and obtain:

$$
\begin{aligned}
D(a, b) & =\{0.6,0.7\}, & D(b, a) & =\{0.3,0.4\}, \\
D(a, c) & =\{1\}\}, & D(c, a) & =\{00\}, \\
D(a, d) & =\{11,1,1\}, & D(d, a) & =\{00,0,0\}, \\
D(b, c) & =\{0.4,0.8\}, & D(c, b) & =\{0.2,0.6\}, \\
D(b, d) & =\{0.4\}, & D(d, b) & =\{0.6\}, \\
D(c, d) & =\{0.6,0.6,0.7\}, & D(d, c) & =\{0.3,0.4,0.4\},
\end{aligned}
$$

Based on the notion of stochastic dominance [20], which is a popular stochastic order for the comparison of probability distributions, we can consider a dominance relation between the preference distributions for two different couples of candidates [27].

Definition 1. Let $\mathscr{C}$ be a set of $k$ candidates, $r$ be the number of voters and $\left(P^{\ell}\right)_{\ell=1}^{r}$ be the partially specified reciprocal relations given by the voters with associated preference distributions $\left\{D\left(a_{i}, a_{j}\right)\right\}_{\left(a_{i}, a_{j}\right) \in \mathscr{C}_{\neq}^{2}}$. For any $\left(a_{i_{1}}, a_{j_{1}}\right),\left(a_{i_{2}}, a_{j_{2}}\right) \in \mathscr{C}_{\neq}^{2}$, we say that $D\left(a_{i_{1}}, a_{j_{1}}\right)$ weakly dominates $D\left(a_{i_{2}}, a_{j_{2}}\right)$, denoted as $D\left(a_{i_{1}}, a_{j_{1}}\right) \unrhd$ $D\left(a_{i_{2}}, a_{j_{2}}\right)$, if, for any $p \in[0,1]$, it holds that

$$
F_{a_{i_{1}}, a_{j_{1}}}^{*}(p) \leq F_{a_{i_{2}}, a_{j_{2}}}^{*}(p),
$$

where, for any $\left(a_{i}, a_{j}\right) \in \mathscr{C}_{\neq}^{2}, F_{a_{i}, a_{j}}^{*}$ is the cumulative preference distribution defined by, for any $p \in[0,1]$ : 


$$
F_{a_{i}, a_{j}}^{*}(p)=\frac{\#\left\{d \in D\left(a_{i}, a_{j}\right) \mid d \leq p\right\}}{\# D\left(a_{i}, a_{j}\right)} .
$$

If $D\left(a_{i_{1}}, a_{j_{1}}\right) \unrhd D\left(a_{i_{2}}, a_{j_{2}}\right)$ and $D\left(a_{i_{2}}, a_{j_{2}}\right) \unrhd D\left(a_{i_{1}}, a_{j_{1}}\right)$, then we say that $D\left(a_{i_{1}}, a_{j_{1}}\right)$ (strictly) dominates $D\left(a_{i_{2}}, a_{j_{2}}\right)$, denoted as $D\left(a_{i_{1}}, a_{j_{1}}\right) \triangleright D\left(a_{i_{2}}, a_{j_{2}}\right)$.

Example 2. We continue with Example 1. Let us consider the preference distributions of $(a, b)$ and $(b, a)$ :

$$
\begin{aligned}
& D(a, b)=\{\{0.6,0.7\}, \\
& D(b, a)=\{\{0.3,0.4\}\} .
\end{aligned}
$$

It is easily verified that $F_{a, b}^{*}$ and $F_{b, a}^{*}$ are respectively given by:

$$
F_{a, b}^{*}(p)= \begin{cases}0 & , \text { if } p \in[0,0.6[ \\ \frac{1}{2} & , \text { if } p \in[0.6,0.7[ \\ 1 & , \text { if } p \in[0.7,1]\end{cases}
$$

and

$$
F_{b, a}^{*}(p)= \begin{cases}0 & , \text { if } p \in[0,0.3[, \\ \frac{1}{2} & , \text { if } p \in[0.3,0.4[, \\ 1 & , \text { if } p \in[0.4,1] .\end{cases}
$$

As illustrated in Fig. 1, we can see that $F_{a, b}^{*}(p) \leq F_{b, a}^{*}(p)$ for any $p \in[0,1]$ with the inequality being strict for $p \in[0.3,0.7[$. We conclude that $D(a, b) \triangleright D(b, a)$ and, thus, $D(b, a) \unrhd D(a, b)$.

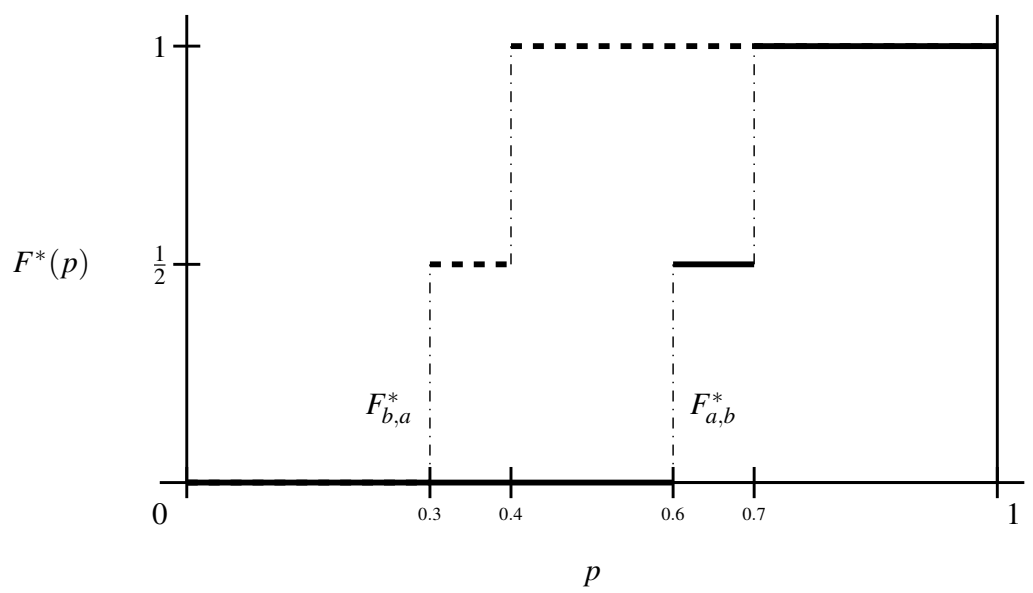

Fig. 1 Graphical representation of $F_{a, b}^{*}$ and $F_{b, a}^{*}$. 
We say that a candidate $a_{i}$ dominates another candidate $a_{j}$ if the preference distribution for $a_{i}$ over $a_{j}$ dominates the preference distribution for $a_{j}$ over $a_{i}$.

Definition 2. Let $\mathscr{C}$ be a set of $k$ candidates, $r$ be the number of voters and $\left(P^{\ell}\right)_{\ell=1}^{r}$ be the partially specified reciprocal relations given by the voters with associated preference distributions $\left\{D\left(a_{i}, a_{j}\right)\right\}_{\left(a_{i}, a_{j}\right) \in \mathscr{C}_{\neq}^{2}}$. We say that a candidate $a_{i} \in \mathscr{C}$ dominates another candidate $a_{j} \in \mathscr{C}$, denoted by $a_{i} \succ a_{j}$, if it holds that

$$
D\left(a_{i}, a_{j}\right) \triangleright D\left(a_{j}, a_{i}\right) .
$$

One should note that neither $\triangleright$ defines a strict order relation on the set of preference distributions, nor $\succ$ defines a strict order relation on the set of candidates. The former is transitive but does not fulfill the antisymmetry property, whereas the latter is antisymmetric (actually asymmetric) but might be cyclical, as can be seen in the following example.

Example 3. We continue with Example 1. As discussed in Example 2, it holds that $D(a, b) \triangleright D(b, a)$, thus, $a \succ b$. In general, $\succ$ is given by:

$$
\succ=\{(a, b),(a, c),(a, d),(b, c),(c, d),(d, b)\} .
$$

We find the cycle $b \succ c, c \succ d$ and $d \succ b$.

\section{Introducing some ideas from the field of social choice theory}

The setting in which each voter expresses his/her partially specified reciprocal relation in the form of a strict order relation (or ranking) on $\mathscr{C}$ has been addressed systematically in the field of social choice theory. In this setting, the dominance $a_{i} \succ a_{j}$ is equivalent to the fact that the number of voters preferring $a_{i}$ over $a_{j}$ is greater than the number of voters preferring $a_{j}$ over $a_{i}$ (for more details, we refer to Section 5 of [27]). The latter fact is commonly referred to as "candidate $a_{i}$ defeats candidate $a_{j}$ by simple majority' $[21,31,17,11]$. It is known that the simple majority relation on the set of candidates might be cyclical. This phenomenon in which all voters express a strict order relation on the set of candidates but the simple majority relation turns out to be cyclical is called 'the Condorcet paradox' or 'the voting paradox' [9]. For this reason, there has been quite some interest in the study of a candidate that defeats all other candidates by simple majority. Such candidate is commonly referred to as the Condorcet candidate. This notion could be easily extended to our setting in which we are dealing with multiple partially specified reciprocal relations.

Definition 3. Let $\mathscr{C}$ be a set of $k$ candidates, $r$ be the number of voters and $\left(P^{\ell}\right)_{\ell=1}^{r}$ be the partially specified reciprocal relations given by the voters with associated preference distributions $\left\{D\left(a_{i}, a_{j}\right)\right\}_{\left(a_{i}, a_{j}\right) \in \mathscr{C}_{\neq}^{2}}$. Consider the dominance relation $\succ$ defined as in Definition 2. A candidate $a_{i} \in \mathscr{C}$ is called the Condorcet winner if, for any $a_{j} \in \mathscr{C} \backslash\left\{a_{i}\right\}$, it holds that $a_{i} \succ a_{j}$. 
Example 4. We continue with Example 1. As discussed in Example 3, it holds that $a \succ b, a \succ c$ and $a \succ d$. Thus, candidate $a$ is the Condorcet winner.

Along the same vein, we could also extend the notions of covering relation [22] and supercovering relation [25] to the setting of multiple partially specified relations.

Definition 4. Let $\mathscr{C}$ be a set of $k$ candidates, $r$ be the number of voters and $\left(P^{\ell}\right)_{\ell=1}^{r}$ be the partially specified reciprocal relations given by the voters with associated preference distributions $\left\{D\left(a_{i}, a_{j}\right)\right\}_{\left(a_{i}, a_{j}\right) \in \mathscr{C}_{\neq}^{2}}$. We say that a candidate $a_{i} \in \mathscr{C}$ covers another candidate $a_{j} \in \mathscr{C}$, denoted by $a_{i} \gg a_{j}$, if it holds that

$$
D\left(a_{i}, a_{j}\right) \triangleright D\left(a_{j}, a_{i}\right),
$$

and, for any $a_{\ell} \in \mathscr{C} \backslash\left\{a_{i}, a_{j}\right\}$,

$$
D\left(a_{j}, a_{\ell}\right) \triangleright D\left(a_{\ell}, a_{j}\right) \Rightarrow D\left(a_{i}, a_{\ell}\right) \triangleright D\left(a_{\ell}, a_{i}\right) .
$$

Definition 5. Let $\mathscr{C}$ be a set of $k$ candidates, $r$ be the number of voters and $\left(P^{\ell}\right)_{\ell=1}^{r}$ be the partially specified reciprocal relations given by the voters with associated preference distributions $\left\{D\left(a_{i}, a_{j}\right)\right\}_{\left(a_{i}, a_{j}\right) \in \mathscr{C}_{\neq}^{2}}$. We say that a candidate $a_{i} \in \mathscr{C}$ supercovers another candidate $a_{j} \in \mathscr{C}$, denoted by $a_{i} \gg a_{j}$, if it holds that

$$
D\left(a_{i}, a_{j}\right) \triangleright D\left(a_{j}, a_{i}\right),
$$

and, for any $a_{\ell} \in \mathscr{C} \backslash\left\{a_{i}, a_{j}\right\}$,

$$
D\left(a_{i}, a_{\ell}\right) \unrhd D\left(a_{j}, a_{\ell}\right) .
$$

As in the original setting of social choice theory, the supercovering relation can be proved to further restrict the covering relation.

Proposition 1. Let $\mathscr{C}$ be a set of $k$ candidates, $r$ be the number of voters and $\left(P^{\ell}\right)_{\ell=1}^{r}$ be the partially specified reciprocal relations given by the voters with associated preference distributions $\left\{D\left(a_{i}, a_{j}\right)\right\}_{\left(a_{i}, a_{j}\right) \in \mathscr{C}_{\neq}^{2}}$. For any two candidates $a_{i}, a_{j} \in \mathscr{C}$, it holds that

$$
a_{i} \gg a_{j} \Rightarrow a_{i} \gg a_{j}
$$

Proof. Consider $a_{i}, a_{j} \in \mathscr{C}$ such that $a_{i} \gg a_{j}$. It trivially holds that $D\left(a_{i}, a_{j}\right) \triangleright$ $D\left(a_{j}, a_{i}\right)$. We only need to prove that, for any $a_{\ell} \in \mathscr{C} \backslash\left\{a_{i}, a_{j}\right\}$,

$$
D\left(a_{j}, a_{\ell}\right) \triangleright D\left(a_{\ell}, a_{j}\right) \Rightarrow D\left(a_{i}, a_{\ell}\right) \triangleright D\left(a_{\ell}, a_{i}\right) .
$$

Consider any $a_{\ell} \in \mathscr{C} \backslash\left\{a_{i}, a_{j}\right\}$ and assume that $D\left(a_{j}, a_{\ell}\right) \triangleright D\left(a_{\ell}, a_{j}\right)$. It thus holds that

$$
D\left(a_{i}, a_{\ell}\right) \unrhd D\left(a_{j}, a_{\ell}\right) \triangleright D\left(a_{\ell}, a_{j}\right) \unrhd D\left(a_{\ell}, a_{i}\right),
$$

and the result finally follows from the transitivity of $\unrhd$ and the definition of $\triangleright$. 
Example 5. We continue with Example 1. Since $a$ is the Condorcet winner and the other three candidates form a majority cycle, the covering relation is given by:

$$
\gg=\{(a, b),(a, c),(a, d)\} .
$$

It thus remains to be seen whether $a$ supercovers any of the other candidates. For $b$, we have that

$$
\begin{aligned}
& D(a, b)=\{\{0.6,0.7\} \triangleright\{\{0.3,0.4\}=D(b, a), \\
& D(a, c)=\{\{1\} \unrhd\{0.4,0.8\}=D(b, c), \\
& D(a, d)=\{\{1,1,1\} \unrhd\{0.4\}=D(b, d) .
\end{aligned}
$$

Thus, it holds that $a \gg b$. Similarly, we have that

$$
\begin{gathered}
D(a, c)=\{\{1\} \triangleright\{\{0\}=D(c, a), \\
D(a, b)=\{\{0.6,0.7\} \unrhd\{\{0.2,0.6\}=D(c, b), \\
D(a, d)=\{\{1,1,1\} \unrhd\{\{0.6,0.6,0.7\}=D(c, d),
\end{gathered}
$$

and

$$
\begin{aligned}
D(a, d)=\{\{1,1,1\} & \triangleright\{\{0,0,0\}=D(d, a), \\
D(a, b)=\{\{0.6,0.7\} & \unrhd\{0.6\}=D(d, b), \\
D(a, c)=\{\{1\} & \unrhd\{\{0.3,0.4,0.4\}=D(d, c) .
\end{aligned}
$$

Thus, it holds that $a \gg c$ and $a \cdot \gg d$. We conclude that

$$
\gg=\{(a, b),(a, c),(a, d)\} .
$$

As anticipated in Proposition 1, it holds that $\gg \subseteq \gg \subseteq \succ$.

Interestingly, unlike the dominance relation $\succ$, the covering relation $\gg$ defines an order relation on $\mathscr{C}$. In the sense of social choice theory, it is known that the Condorcet winner is equivalently defined by using the covering relation rather than the dominance relation. This result is also immediately extended to the setting of multiple partially specified reciprocal relations.

Proposition 2. Let $\mathscr{C}$ be a set of $k$ candidates, $r$ be the number of voters and $\left(P^{\ell}\right)_{\ell=1}^{r}$ be the partially specified reciprocal relations given by the voters with associated

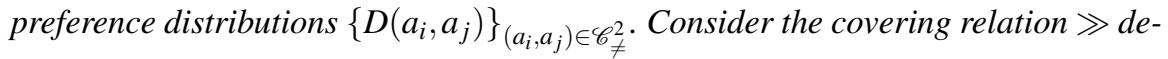
fined as in Definition 4. A candidate $a_{i} \in \mathscr{C}$ is the Condorcet winner if and only if, for any $a_{j} \in \mathscr{C} \backslash\left\{a_{i}\right\}$, it holds that $a_{i} \gg a_{j}$.

Proof. On the one hand, let $a_{i}$ be the Condorcet winner. Consider any $a_{\ell} \in \mathscr{C} \backslash\left\{a_{i}, a_{j}\right\}$ such that $a_{j} \succ a_{\ell}$. By definition, it holds that $a_{i} \succ a_{j}$ for any $a_{j} \in \mathscr{C} \backslash\left\{a_{i}\right\}$, thus, $a_{i} \succ a_{\ell}$. We conclude that $a_{i} \gg a_{j}$. 
On the other hand, let $a_{i}$ be such that $a_{i} \gg a_{j}$ for any $a_{j} \in \mathscr{C} \backslash\left\{a_{i}\right\}$. By definition of $\gg$, it follows that $a_{i} \succ a_{j}$ for any $a_{j} \in \mathscr{C} \backslash\left\{a_{i}\right\}$, which exactly is the definition of a Condorcet winner.

Unlike with the dominance relation $\succ$ and the covering relation $\gg$, requiring a candidate to supercover all other candidates yields a stronger type of winner than the Condorcet winner: the pairwise winner [25].

Definition 6. Let $\mathscr{C}$ be a set of $k$ candidates, $r$ be the number of voters and $\left(P^{\ell}\right)_{\ell=1}^{r}$ be the partially specified reciprocal relations given by the voters with associated preference distributions $\left\{D\left(a_{i}, a_{j}\right)\right\}_{\left(a_{i}, a_{j}\right) \in \mathscr{C}_{\ddagger}^{2}}$. Consider the supercovering relation $\gg$ defined as in Definition 5. A candidate $a_{i} \in \mathscr{C}$ is called the pairwise winner if, for any $a_{j} \in \mathscr{C} \backslash\left\{a_{i}\right\}$, it holds that $a_{i} \gg a_{j}$.

Obviously, if a pairwise winner exists, a Condorcet winner exists and needs to coincide with this pairwise winner.

Example 6. We continue with Example 1. From Example 5, we conclude that $a$ is both the Condorcet winner and the pairwise winner.

\section{Conclusions}

In this contribution, we have brought the notions of dominance relation, covering relation and supercovering relation to the field of preference modelling. In particular, we have adapted these three notions to the setting in which each voter provides a partially specified reciprocal relation. There is a clear analogy between this setting and the one in social choice theory due to the preorder nature of the considered dominance relation on the set of preference distributions for couples of candidates.

Future research is anticipated in two directions. Firstly, one could follow the direction in [27] and study how to act in case the covering and supercovering relations are sparse and, thus, there does not exist a pairwise/Condorcet winner. Secondly, one could study the notions of Borda-dominance relation [12] and superdominance relation [26], which relate to the positionalistic point of view of social choice theory (usually attributed to Borda [6]), in the context of multiple partially specified reciprocal relations, following a similar direction to that of $[15,16]$.

\section{Acknowledgements}

This research has been partially supported by Spanish MINECO (TIN2017-87600P). Raúl Pérez-Fernández acknowledges the support of the Research Foundation of Flanders (FWO17/PDO/160). 


\section{References}

1. K. J. Arrow. A difficulty in the concept of social welfare. The Journal of Political Economy, 58(4):328-346, 1950.

2. K. J. Arrow. Social Choice and Individual Values. Yale University Press, New Haven, second edition, 1963.

3. M. Balinski and R. Laraki. A theory of measuring, electing and ranking. PNAS, 104(21):87208725, 2007.

4. M. Balinski and R. Laraki. Majority Judgment: Measuring, Ranking, and Electing. MIT press, Cambridge, 2010.

5. J. P. Barthelemy and B. Monjardet. The median procedure in cluster analysis and social choice theory. Mathematical Social Sciences, 1:235-267, 1981.

6. J. C. Borda. Mémoire sur les Élections au Scrutin. Histoire de l'Académie Royale des Sciences, Paris, 1781.

7. F. Chiclana, F. Herrera, and E. Herrera-Viedma. Integrating three representation models in fuzzy multipurpose decision making based on fuzzy preference relations. Fuzzy Sets and Systems, 97(1):33-48, 1998.

8. F. Chiclana, F. Herrera, and E. Herrera-Viedma. Integrating multiplicative preference relations in a multipurpose decision-making model based on fuzzy preference relations. Fuzzy Sets and Systems, 122:277-291, 2001.

9. M. Condorcet. Essai sur l'Application de l'Analyse à la Probabilité des Décisions Rendues à la Pluralité des Voix. De l'Imprimerie Royale, Paris, 1785.

10. B. De Baets, H. De Meyer, B. De Schuymer, and S. Jenei. Cyclic evaluation of transitivity of reciprocal relations. Social Choice and Welfare, 26(2):217-238, 2006.

11. P. C. Fishburn. Conditions for simple majority decision functions with intransitive individual indifference. Journal of Economic Theory, 2:354-367, 1970.

12. P. C. Fishburn. Paradoxes of voting. The American Political Science Review, 68(2):537-546, 1974.

13. J. C. Fodor and M. R. Roubens. Fuzzy Preference Modelling and Multicriteria Decision Support. Kluwer Academic Publishers, Dordrecht, 1994.

14. J. L. García-Lapresta. A general class of simple majority decision rules based on linguistic opinions. Information Sciences, 176:352-365, 2006.

15. J. L. García-Lapresta and M. Martínez-Panero. Borda counts versus approval voting: A fuzzy approach. Public Choice, 112:167-184, 2002.

16. J. L. García-Lapresta, M. Martínez-Panero, and L. C. Meneses. Defining the Borda count in a linguistic decision making context. Information Sciences, 179:2309-2316, 2009.

17. K. Inada. The simple majority decision rule. Econometrica, 37(3):490-506, 1969.

18. J. Kacprzyk, H. Nurmi, and M. Fedrizzi. Consensus under Fuzziness, volume 10. Kluwer Academic, Boston, 1996.

19. J. G. Kemeny. Mathematics without numbers. Daedalus, 88(4):577-591, 1959.

20. H. Levy. Stochastic Dominance: Investment Decision Making under Uncertainty. Springer, Berlin, third edition, 2016.

21. K. O. May. A set of independent necessary and sufficient conditions for simple majority decision. Econometrica, 20:680-684, 1952.

22. N. R. Miller. A new solution set for tournaments and majority voting: further graph-theoretical approaches to the theory of voting. American Journal of Political Science, 24(1):68-96, 1980.

23. J. F. Nash. The bargaining problem. Econometrica, 28:155-162, 1950.

24. H. T. Nguyen, O. Kosheleva, and V. Kreinovich. Decision making beyond Arrow's "impossibility theorem," with the analysis of effects of collusion and mutual attraction. International Journal of Intelligent Systems, 24:27-47, 2008.

25. R. Pérez-Fernández and B. De Baets. The supercovering relation, the pairwise winner, and more missing links between Borda and Condorcet. Social Choice and Welfare, 50:329-352, 2018. 
26. R. Pérez-Fernández and B. De Baets. The superdominance relation, the positional winner, and more missing links between borda and condorcet. Journal of Theoretical Politics, 31(1):4665, 2019.

27. R. Pérez-Fernández, M. Rademaker, P. Alonso, I. Díaz, S. Montes, and B. De Baets. Monotonicity-based ranking on the basis of multiple partially specified reciprocal relations. Fuzzy Sets and Systems, 325:69-96, 2017.

28. M. Roubens. Choice procedures in fuzzy multicriteria decision analysis based on pairwise comparisons. Fuzzy Sets and Systems, 84:135-142, 1996.

29. M. Roubens and P. Vincke. Preference modelling. Springer Verlag, Berlin, 1985.

30. M. Schulze. A new monotonic, clone-independent, reversal symmetric, and Condorcetconsistent single-winner election method. Social Choice and Welfare, 36:267-303, 2011.

31. A. K. Sen. A possibility theorem on majority decisions. Econometrica, 34(2):491-499, 1966.

32. A. K. Sen. Collective Choice and Social Welfare. Holden-Day, San Francisco, 1970.

33. T. N. Tideman. Independence of clones as a criterion for voting rules. Social Choice and Welfare, 4(3):185-206, 1987.

34. H. P. Young. Condorcet's theory of voting. American Political Science Review, 82(4):1231$1244,1988$.

35. T. M. Zavist and T. N. Tideman. Complete independence of clones in the ranked pairs rule. Social Choice and Welfare, 6(2):167-173, 1989. 